\title{
Ivermectin does not protect against SARS-CoV-2 infection in the Syrian hamster model
}

1 KU Leuven Department of Microbiology, Immunology and Transplantation, Rega Institute for Medical Research, Laboratory of Virology and Chemotherapy, B-3000 Leuven, Belgium.

2 Global Virus Network, GVN.

3 KU Leuven Department of Imaging and Pathology, Translational Cell and Tissue Research, B-3000 Leuven, Belgium; Division of Translational Cell and Tissue Research.

\#C.S.F and R.A. contributed equally to that work

* Correspondence: Prof. Johan Neyts. Email: johan.neyts@kuleuven.be.

\begin{abstract}
Ivermectin, an FDA-approved antiparasitic drug, has been reported to have in vitro activity against SARS-CoV-2. An increasing off-label use of Ivermectin for COVID-19 has been reported. We here assessed the effect of Ivermectin in Syrian hamsters infected with the SARS-CoV-2 Beta (B.1.351) variant. Infected animals received a clinically relevant dose of Ivermectin $(0.4 \mathrm{mg} / \mathrm{kg}$ subcutaneously dosed) once daily for four consecutive days after which the effect was quantified. Ivermectin monotherapy did not reduce lung viral load and even significantly worsened the SARS-CoV-2-induced lung pathology. Additionally, it did not potentiate the activity of Molnupiravir (Lagevrio ${ }^{\mathrm{TM}}$ ) when combined with this drug. This study contributes to the growing body of evidence that Ivermectin does not result in a beneficial effect in the treatment of COVID-19. These findings are important given the increasing, dangerous off-label use of Ivermectin for the treatment of COVID-19.
\end{abstract}

Keywords: COVID-19; Ivermectin; Molnupiravir; off-label use; hamster model

\section{Main text}

Two years after it was declared a global pandemic by the World Health Organization, the coronavirus disease (COVID-19) continues its devastating impact, having claimed officially over 5.9 million lives as of $22^{\text {nd }} \mathrm{Feb} 2022$ [1]. The etiological agent, the severe acute respiratory syndrome coronavirus 2 (SARS-CoV-2), first emerged in Wuhan, China in December 2019 and has since evolved into variants emerging from different regions of the world, namely the Alpha, Beta, Delta, and more recently Omicron variants of concern (VoC) $[2,3]$. These $\mathrm{VoC}$ have been implicated in resurgence of infections and mortality, with increased transmissibility and potential escape from both vaccine- and natural infection-induced immunity [3-5].

Ivermectin is an FDA-approved, broad-spectrum antiparasitic drug with a range of other activities, including antiviral and host immunomodulation effects [6]. This widely-accessible drug has been attracting increasing off-label use for COVID-19 since its in vitro activity against SARS-CoV-2 was reported [7], which is of growing concern. In an in vivo model of mouse hepatitis virus, a type 2 family RNA coronavirus similar to SARS-CoV-2, Ivermectin reduced the viral load and protected mice from disease [8]. In Syrian hamsters infected with SARS-CoV-2, treatment with Ivermectin did not result in a direct antiviral activity, but rather apparently showed some immunomodulatory effects, improved lung pathology and clinical presentations [9]. Ivermectin is being assessed as a treatment for COVID-19 in a large number of clinical trials, with no clear evidence of its clinical benefit so far [10].

Molnupiravir (Lagevrio ${ }^{\mathrm{TM}}$, EIDD-2801) is the orally bioavailable counterpart of the ribonucleoside analogue N4-hydroxycytidine (NHC, EIDD-1931), which was initially developed for the treatment of influenza [11]. NHC exerts a broad-spectrum antiviral activity against multiple RNA viruses of different families by its incorporation into viral RNA, resulting in the accumulation of deleterious mutations in the nascent viral RNA, and consequently, error ca- 
tastrophe [12]. In pre-clinical infection models in mice, Syrian hamsters, and ferrets, Molnupiravir demonstrated efficacy against SARS-CoV-2 [13-15], including against several VoC [16]. The drug has been approved in many regions for treatment of COVID-19.

Here, we evaluate the antiviral efficacy of Ivermectin against SARS-CoV-2 Beta (B.1.351) VoC in a hamster infection model alone or in combination with Molnupiravir; this combination was inspired by the observation of a pronounced combined activity of Molnupiravir and Favipiravir [17].

To that end, we tested the efficacy of single treatment with Ivermectin $(0.4 \mathrm{mg} / \mathrm{kg}$, subcutaneous, once daily (QD)) or Molnupiravir (150 mg/kg, oral BID) or the combination of both. Briefly, 6-8 weeks female SG hamsters were treated with the intended dose of each compound or the vehicle (i.e. the control group) for four consecutive days starting one hour before intranasal infection with $1 \times 10^{4}$ TCID 50 of SARS-CoV-2 Beta (B.1.351) variant Beta B.1.351 (derived from hCoV-19/Belgium/rega-1920/2021; EPI_ISL_896474, 2021-01-11) [18] (Figure 1A). At day 4 post-infection (pi), the animals were euthanized and organs were collected for quantification of viral RNA, infectious virus titers and lung histopathology as described previously [19] (Figure 1A). Monotherapy with Ivermectin (0.4 mg/kg, once daily) did not result in any significant reduction of viral RNA (Figure 1B) or infectious virus titers (Figure 1C) in the lung of treated-hamsters as compared to the vehicle control. On the other hand, single treatment with Molnupiravir (150 mg/kg BID) significantly reduced viral RNA and infectious virus titers in the lungs by $1.1(\mathrm{P}=0.0048)$ and $1.8(\mathrm{P}=0.0005) \log _{10} / \mathrm{mg}$ lung tissue, respectively (Figure 1B/C). Treatment of infected hamsters with a combination of Molnupiravir (150 mg/kg, BID) and Ivermectin $(0.4 \mathrm{mg} / \mathrm{kg}$, once daily) resulted in a reduction of the lung viral RNA loads by $1.1 \log _{10}(\mathrm{P}=0.022$, Figure $1 \mathrm{~B})$ and lung infectious virus titers by 1.9 $(\mathrm{P}=0.0002$, Figure $1 \mathrm{C}) \log _{10} / \mathrm{mg}$ lung tissue, which is similar in potency to the single treatment with Molnupiravir ( $150 \mathrm{mg} / \mathrm{kg}$, BID). No significant weight loss or toxicity signs were observed in any of the treated groups (Figure 1D). However, the average \%body weight change on the day of the sacrifice (compared to day zero) in the Ivermectin single treatment group (average of $-1.7 \%$ ) was markedly lower than the vehicle-treated group (average of 2.6\%), Figure 1D.

The median histopathology score in the single Ivermectin treatment group (5.8) was significantly higher than the vehicle treated control (median score of 4.5, P=0.04) (Figure 2A). A significant improvement in lung pathology was observed in the single Molnupiravir-treated group (median score of $3.5, \mathrm{P}=0.04$ ) while the combined Molnupiravir/Ivermectin treatment resulted in a median histopathology score of 3.8 (Figure 2A). Hematoxylin/eosin (H\&E)stained images of lungs of the Ivermectin-treated hamsters revealed severe peri-vascular inflammation with vasculitis and significant multifocal bronchopneumonia (Figure 2B). On the other hand, the lungs of animals treated with Molnupiravir as a single treatment showed very limited peribronchial and peri-vascular (Figure 2B).

In our study, Ivermectin did not result in any antiviral activity against SARS-CoV-2 in Syrian hamsters as monotherapy. This is consistent with a previous study published as well in hamsters, whereby Ivermectin was dosed once subcutaneously at $0.4 \mathrm{mg} / \mathrm{kg}$ (an antiparasitic dose used in the clinical setting) and monitored for four days [9]. However, unlike the previous study, we did not notice an improvement in SARS-CoV-2-induced lung pathology with Ivermectin. Transcriptomic profiling of the lungs was performed in the previous study to compare expression levels of inflammatory genes with vehicle and Ivermectin treatment, as well as a descriptive histopathological analysis which indicated that SARS-CoV-2-infected, Ivermectin-treated hamsters exhibited reduced degrees of edema and congestion, with greater numbers of mononuclear cells in the alveolar spaces [9]. In our study, a cumulative lung score was performed based on ten different parameters, including edema and congestion, which had similar scorings in both vehicle-treated and Ivermectin-treated groups. Additionally, no mononuclear cells in the alveolar spaces were observed. These differences in findings may be due to slight hamster age differences or virus strain differences used for infection, namely the SARS-CoV-2 Beta variant was used in our study and the ancestral SARSCoV-2 strain (BetaCoV/France/IDF00372/2020) was used in the previous study [9].

Additionally, Ivermectin did not potentiate the activity of Molnupiravir in our hands, indicating that it is unlikely to have a significant role in directly or indirectly modulating SARS-CoV- 
2 replication. $\mathrm{A} \mathrm{C}_{\max }$ of $80.2 \mathrm{ng} / \mathrm{ml}$ was measured in Syrian hamsters when treated once subcutaneously with $0.4 \mathrm{mg} / \mathrm{kg}$ of Ivermectin [20], comparable to that measured in humans when dosed at similar levels orally $[9,21]$. Taken together with other available evidence, it suggests that in order to achieve therapeutic activity against SARS-CoV-2 (IC 50 of $1750 \mathrm{ng} / \mathrm{ml}$ [7]), the dosing levels of Ivermectin in humans would need to be substantially increased from the approved clinical dose to potentially toxic doses [22-24]. This is in line with the fact that several studies claiming the clinical benefits of Ivermectin in COVID-19 have been found to be flawed [25], and larger, better designed trials such as the TOGETHER trial (NCT0472742) have demonstrated that Ivermectin does not have clinical efficacy against COVID-19 [26,27].

In conclusion, we report that Ivermectin, at a clinically relevant dose, does not reduce lung viral load nor improve lung pathology in Syrian hamsters infected with SARS-CoV-2 Beta variant. In fact, virus-induced lung pathology increases in severity in Ivermectin treated animals. Moreover, the drug does not potentiate the antiviral activity of Molnupiravir (Lagevrio ${ }^{\mathrm{TM}}$ ). This study contributes to the growing body of evidence that Ivermectin does not results in a clinical beneficial effect in the treatment of COVID-19 at the current approved dose. Hence, we strongly advice against the increasing, dangerous off-label use of Ivermectin for the treatment of COVID-19.

105 106 107 108 109 110 111 112 


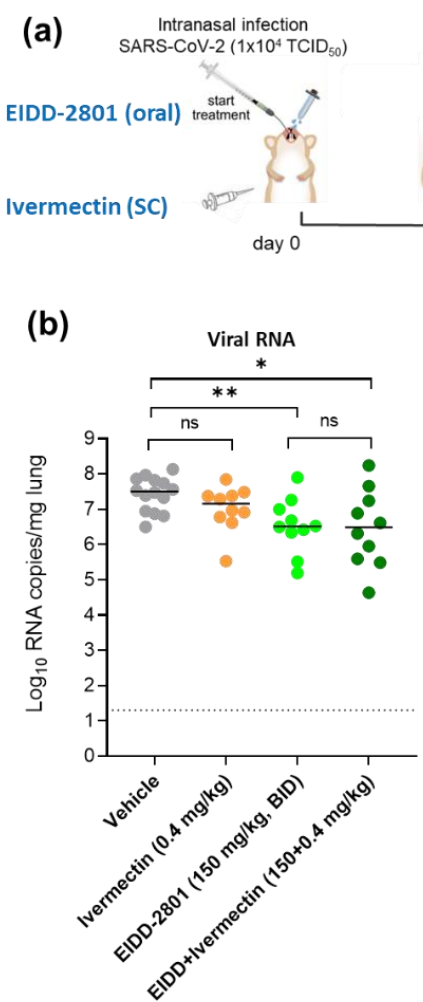

(c) Infectious virus titers

(d)

Lung samples on day 4 to determine:

- Infectious virus

- Viral RNA (genomic)

- Histopathology

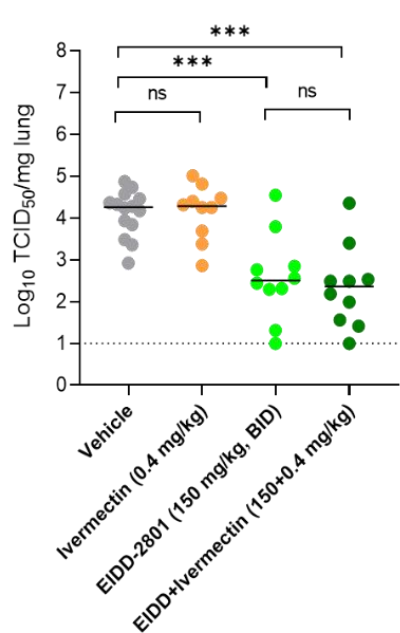

Body weight change

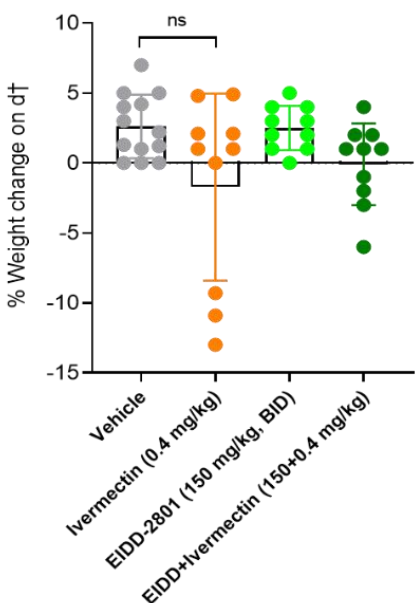

Figure 1. Antiviral efficacy of Ivermectin as a single or combination therapy against SARS-CoV-2 beta (B.1.351) variant in a hamster infection model. (a) Set-up of the study. (b) Viral RNA levels in the lungs of control (vehicle-treated), Ivermectin-treated $(0.4 \mathrm{mg} / \mathrm{kg}, \mathrm{QD})$, EIDD-2801-treated (150 $\mathrm{mg} / \mathrm{kg}$, BID) and combination-treated (EIDD-2801+Ivermectin at $150 \mathrm{mg} / \mathrm{kg}$ BID+0.4 mg/kg QD) SARSCoV-2 (B.1.351)-infected hamsters at day 4 post-infection (pi) are expressed as log10 SARS-CoV-2 RNA copies per mg lung tissue. Individual data and median values are presented. (c) Infectious viral loads in the lungs of control (vehicle-treated), Ivermectin-treated, EIDD-2801-treated and combination-treated (EIDD-2801+Ivermectin) SARS-CoV-2-infected hamsters at day 4 pi are expressed as log10 TCID50 per mg lung tissue. Individual data and median values are presented. (d) Weight change at day 4 pi in percentage, normalized to the body weight at the time of infection. Bars represent means \pm SD. Data were analyzed with the Mann-Whitney $U$ test. ${ }^{*} \mathrm{P}<0.05,{ }^{* *} \mathrm{P}<0.01,{ }^{* * *} \mathrm{P}<0.001$, ns=non-significant. EIDD=EIDD-2801. All data (panels b, c, d) are from two independent experiments with $\mathrm{n}=14$ for vehicle group and $\mathrm{n}=10$ for the other groups. 
bioRxiv preprint doi: https://doi.org/10.1101/2022.02.22.481472; this version posted February 24, 2022. The copyright holder for this

(a)

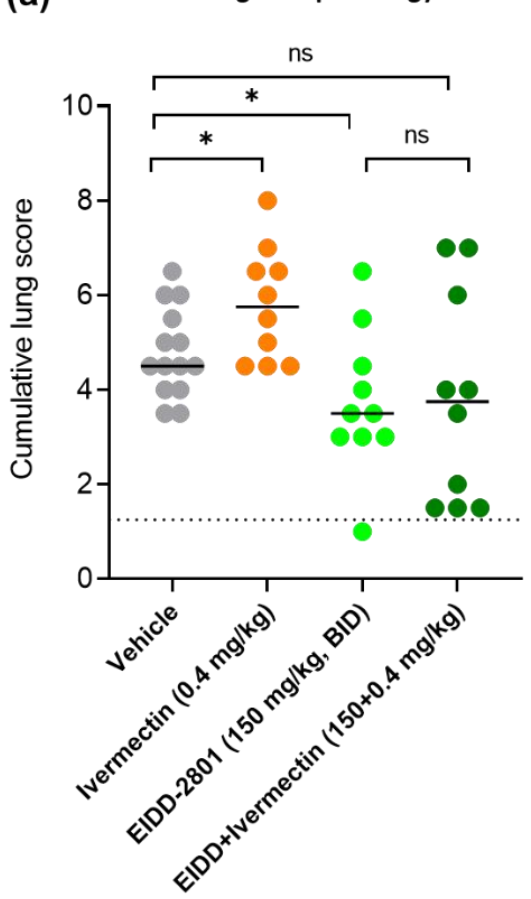

(b) Vehicle-treated
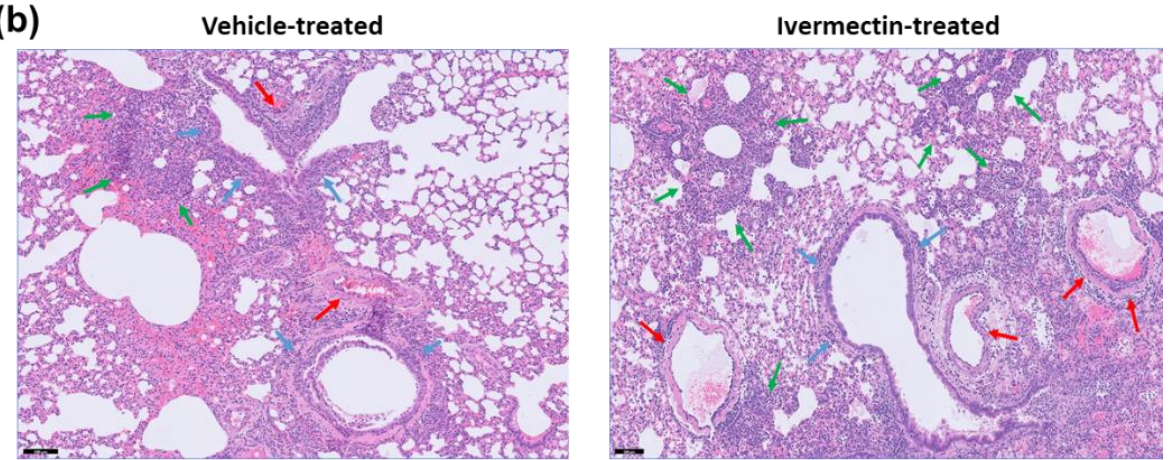

EIDD-2801-treated

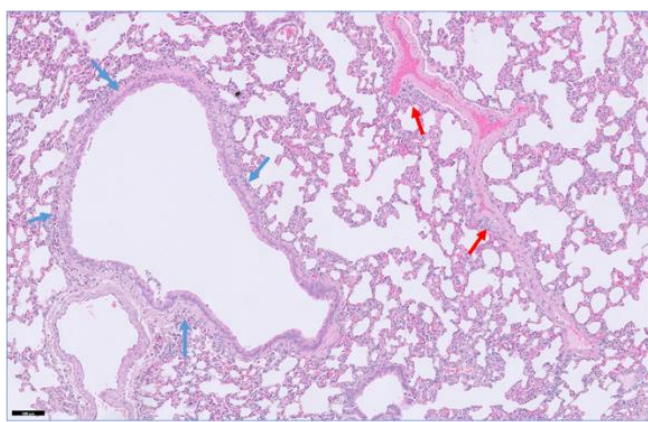

Figure 2. Lung histopathology of SARS-CoV-2-infected Syrian hamsters following treatment with Ivermectin as a single or combination therapy. (a) Cumulative severity score from H\&E stained slides of lungs from control (vehicle-treated Ivermectin-treated $(0.4 \mathrm{mg} / \mathrm{kg}, \mathrm{QD})$, EIDD-2801-treated

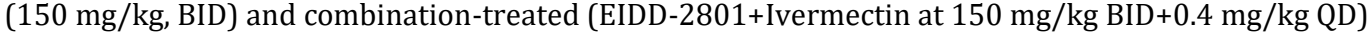
SARS-CoV-2 (B.1.351)-infected hamsters at day 4 post-infection (pi). Individual data and median values are presented and the dotted line represents the median score of untreated non-infected hamsters. Data were analyzed with the Mann-Whitney $\mathrm{U}$ test. ${ }^{*} \mathrm{P}<0.05$, ns=non-significant. Data are from two independent experiments with $n=14$ for vehicle group and $n=10$ for the other groups. EIDD=EIDD-2801. (b) Representative H\&E images of lungs of control (vehicle-treated), Ivermectin-treated and EIDD-2801treated SARS-CoV-2 (B.1.351)-infected hamsters at day 4 post-infection (pi). Lung from vehicle control group showed peribronchial inflammation with intra-bronchial cell debris (blue arrows), perivascular inflammation with endothelialitis (red arrows) and bronchopneumonia (green arrows) surrounded by intra-alveolar hemorrhage. Image of the lung from Ivermectin-treated animal showed severe peri-vascular inflammation with vasculitis (red arrows), limited and focal peri-bronchial inflammation (blue arrows) and significant and multifocal bronchopneumonia (green arrows). On the other hand, lung from Molnupiravir (EIDD-2801) revealed very limited peribronchial (blue arrows) and peri-vascular (red arrows) inflammation. Scale bar $100 \mu \mathrm{M}$.

Author Contributions: Conceptualization, R.A., C.S.F. and J.N.; methodology, R.A. and C.S.F.; formal analysis, C.S.F., B.W. and R.A.; data curation, R.A.; validation, R.A. C.S.F. and J.N.; writing-original draft preparation, R.A. and C.S.F.; writing-review and editing, R.A. and J.N.; visualization, R.A. and B.W.; supervision, C.S.F. and R.A. and J.N.; project administration, R.A. and C.S.F; resources, S.D.J, J.N.; funding acquisition, L.V., D.J. and J.N. All authors have read and agreed to the published version of the manuscript.

Funding: This project has received funding from the Covid-19-Fund KU Leuven/UZ Leuven and the COVID-19 call of FWO (G0G4820N), the European Union's Horizon 2020 research and innovation program under grant agreements No 101003627 (SCORE project) and Bill \& Melinda Gates Foundation (BGMF) under grant agreement INV-006366. This work also has been done under the CARE project, which has received funding from the Innovative Medicines Initiative 2 Joint Undertaking (JU) under grant agreement No 101005077. The JU receives support from the European Union's Horizon 2020 research and innovation programme and EFPIA and BILL \& MELINDA GATES FOUNDATION, GLOBAL HEALTH DRUG DISCOVERY INSTITUTE, UNIVERSITY OF DUNDEE. The content of this publication only reflects the author's view and the JU is not responsible for any use that may be made of the information it contains.

Data Availability Statement: All of the data generated or analyzed during this study are included in this published article.

\section{1}


Acknowledgments: We thank Carolien De Keyzer, Lindsey Bervoets, Thibault Francken, Stijn Hendrickx, Niels Cremers for excellent technical assistance. We are grateful to Piet Maes for kindly providing the SARS-CoV-2 strain used in this study. We thank Prof. Jef Arnout and Dr. Annelies Sterckx (KU Leuven Faculty of Medicine, Biomedical Sciences Group Management) and Animalia and Biosafety Departments of KU Leuven for facilitating the animal studies. We thank the Histology department of KU Leuven for technical support for histopathological analyses. We also thank Fran Berlioz-Seux, Rob Jordan and Betsy Russell for helpful discussion.

Conflicts of Interest: The authors declare no conflict of interest. The funders had no role in the design of the study; in the collection, analyses, or interpretation of data; in the writing of the manuscript, or in the decision to publish the results. 


\section{References}

1. WHO Coronavirus (COVID-19) Dashboard.

2. Karim, S.S.A.; Karim, Q.A. Omicron SARS-CoV-2 variant: a new chapter in the COVID-19 pandemic. Lancet 2021, 398, $2126-2128$.

3. Plante, J.A.; Mitchell, B.M.; Plante, K.S.; Debbink, K.; Weaver, S.C.; Menachery, V.D. The variant gambit: COVID-19’s next move. Cell Host Microbe 2021, 29, 508-515.

4. $\quad$ Davies, N.G.; Abbott, S.; Barnard, R.C.; Jarvis, C.I.; Kucharski, A.J.; Munday, J.D.; Pearson, C.A.B.; Russell, T.W.; Tully, D.C.; Washburne, A.D.; et al. Estimated transmissibility and impact of SARS-CoV-2 lineage B.1.1.7 in England. Science (80-. ). 2021, 372.

5. Wang, P.; Nair, M.S.; Liu, L.; Iketani, S.; Luo, Y.; Guo, Y.; Wang, M.; Yu, J.; Zhang, B.; Kwong, P.D.; et al. Antibody resistance of SARSCoV-2 variants B.1.351 and B.1.1.7. Nature 2021, 593, 130-135.

6. Heidary, F.; Gharebaghi, R. Ivermectin: a systematic review from antiviral effects to COVID-19 complementary regimen. J. Antibiot. (Tokyo). 2020, 73, 593-602.

7. Caly, L.; Druce, J.D.; Catton, M.G.; Jans, D.A.; Wagstaff, K.M. The FDA-approved drug ivermectin inhibits the replication of SARSCoV-2 in vitro. Antiviral Res. 2020, 178, 104787

8. Arévalo, A.P.; Pagotto, R.; Pórfido, J.L.; Daghero, H.; Segovia, M.; Yamasaki, K.; Varela, B.; Hill, M.; Verdes, J.M.; Duhalde Vega, M.; et al. Ivermectin reduces in vivo coronavirus infection in a mouse experimental model. Sci. Rep. 2021, 11, 7132.

9. de Melo, G.D.; Lazarini, F.; Larrous, F.; Feige, L.; Kornobis, E.; Levallois, S.; Marchio, A.; Kergoat, L.; Hardy, D.; Cokelaer, T.; et al. Attenuation of clinical and immunological outcomes during SARS-CoV-2 infection by ivermectin. EMBO Mol. Med. 2021, 13, e14122.

10. Table: Ivermectin Clinical Data.

11. Toots, M.; Yoon, J.J.; Cox, R.M.; Hart, M.; Sticher, Z.M.; Makhsous, N.; Plesker, R.; Barrena, A.H.; Reddy, P.G.; Mitchell, D.G.; et al. Characterization of orally efficacious influenza drug with high resistance barrier in ferrets and human airway epithelia. Sci. Transl. Med. 2019, 11.

12. Urakova, N.; Kuznetsova, V.; Crossman, D.K.; Sokratian, A.; Guthrie, D.B.; Kolykhalov, A.A.; Lockwood, M.A.; Natchus, M.G.; Crowley, M.R.; Painter, G.R.; et al. $\quad$ B-D-N 4 -Hydroxycytidine Is a Potent Anti-alphavirus Compound That Induces a High Level of Mutations in the Viral Genome. J. Virol. 2017, 92.

13. Rosenke, K.; Hansen, F.; Schwarz, B.; Feldmann, F.; Haddock, E.; Rosenke, R.; Barbian, K.; Meade-White, K.; Okumura, A.; Leventhal, S.; et al. Orally delivered MK-4482 inhibits SARS-CoV-2 replication in the Syrian hamster model. Nat. Commun. $2021,12$.

14. Wahl, A.; Gralinski, L.E.; Johnson, C.E.; Yao, W.; Kovarova, M.; Dinnon, K.H.; Liu, H.; Madden, V.J.; Krzystek, H.M.; De, C.; et al. SARSCoV-2 infection is effectively treated and prevented by EIDD-2801. Nature 2021, 591, 451-457.

15. Cox, R.M.; Wolf, J.D.; Plemper, R.K. Therapeutically administered ribonucleoside analogue MK-4482/EIDD-2801 blocks SARS-CoV2 transmission in ferrets. Nat. Microbiol. 2020.

16. Abdelnabi, R.; Foo, C.S.; De Jonghe, S.; Maes, P.; Weynand, B.; Neyts, J. Molnupiravir Inhibits Replication of the Emerging SARSCoV-2 Variants of Concern in a Hamster Infection Model. J. Infect. Dis. 2021, 224, 749-753.

17. Abdelnabi, R.; Foo, C.S.; Kaptein, S.J.F.; Zhang, X.; Do, T.N.D.; Langendries, L.; Vangeel, L.; Breuer, J.; Pang, J.; Williams, R.; et al. The combined treatment of Molnupiravir and Favipiravir results in a potentiation of antiviral efficacy in a SARS-CoV-2 hamster infection model. EBioMedicine 2021, 72.

18. Abdelnabi, R.; Boudewijns, R.; Foo, C.S.; Seldeslachts, L.; Sanchez-Felipe, L.; Zhang, X.; Delang, L.; Maes, P.; Kaptein, S.J.F.; Weynand, B.; et al. Comparing infectivity and virulence of emerging SARS-CoV-2 variants in Syrian hamsters. EBioMedicine 2021, 68, 103403. 19. Kaptein, S.J.F.; Jacobs, S.; Langendries, L.; Seldeslachts, L.; ter Horst, S.; Liesenborghs, L.; Hens, B.; Vergote, V.; Heylen, E.; Barthelemy, K.; et al. Favipiravir at high doses has potent antiviral activity in SARS-CoV-2-infected hamsters, whereas hydroxychloroquine lacks activity. Proc. Natl. Acad. Sci. U. S. A. 2020, 117, 26955-26965. 
ivermectin on blood-feeding Phlebotomus papatasi, and the promastigote stage of Leishmania major. Vector Borne Zoonotic Dis.

2011, 11, 43-52.

21. Guzzo, C.A.; Furtek, C.I.; Porras, A.G.; Chen, C.; Tipping, R.; Clineschmidt, C.M.; Sciberras, D.G.; Hsieh, J.Y.K.; Lasseter, K.C. Safety, tolerability, and pharmacokinetics of escalating high doses of ivermectin in healthy adult subjects. J. Clin. Pharmacol. 2002, 42, $1122-1133$.

22. Chaccour, C.; Hammann, F.; Ramón-García, S.; Rabinovich, N.R. Ivermectin and COVID-19: Keeping Rigor in Times of Urgency. Am. J. Trop. Med. Hyg. 2020, 102, 1156-1157.

23. Jermain, B.; Hanafin, P.O.; Cao, Y.; Lifschitz, A.; Lanusse, C.; Rao, G.G. Development of a Minimal Physiologically-Based Pharmacokinetic Model to Simulate Lung Exposure in Humans Following Oral Administration of Ivermectin for COVID-19 Drug Repurposing. J. Pharm. Sci. 2020, 109, 3574-3578.

24. Schmith, V.D.; Zhou, J.J.; Lohmer, L.R.L. The Approved Dose of Ivermectin Alone is not the Ideal Dose for the Treatment of COVID19. Clin. Pharmacol. Ther. 2020, 108, 762-765.

25. Lawrence, J.M.; Meyerowitz-Katz, G.; Heathers, J.A.J.; Brown, N.J.L.; Sheldrick, K.A. The lesson of ivermectin: meta-analyses based on summary data alone are inherently unreliable. Nat. Med. 2021, 27, 1853-1854. 